\title{
An Overview of Spectrum Sensing and its Techniques
}

\author{
${ }^{1}$ Munpreet Kaur Raina, ${ }^{2}$ Gagangeet Singh Aujla \\ ${ }^{I}$ Computer Science and Engineering Chandigarh Engineering College Landran, Mohali. \\ ${ }^{2}$ Assistant Professor, Computer Science and Engineering, Chandigarh Engineering College Landran, Mohali.
}

\begin{abstract}
Cognitive radio networks provide high bandwidth to mobile users via heterogeneous wireless architectures and dynamic spectrum access techniques. It basically avoids congestion in wireless communication by exploiting unused radio spectrum that is not significantly engaged through a licensed user. This is the most promising and efficient technique for the proper utilization of each and every frequency band. In this paper a brief introduction of different sensing techniques has been given and also the various aspects related to these techniques. Different issues related to spectrum sensing techniques and their comparison has been done in the paper.
\end{abstract}

Index Terms: Cognitive radio, Primary user, Secondary user, Spectrum sensing, Signal processing techniques.

\section{Introduction}

The idea of Cognitive radio was first conceived by Sir Joseph Mitola at the Royal Institute of Technology in the year 1998. The leading factor behind this was increasing need for the radio spectrum. The improved communication speed was the unusual drive and this was satisfied by effectively utilizing the radio spectrum [1]. Due to static allocation of spectrum, the allotted spectrum was not properly utilized. It has become most difficult to find vacant bands either to set up a new service or to enhance the existing one. In order to overcome these problems we are going for "Dynamic Spectrum Management" which improves the utilization of spectrum. Cognitive Radio performs on this dynamic Spectrum Management principle which solves the issue of spectrum underutilization in wireless communication in an efficient manner. It is reasonable because it is well informed of its environment and adapt to the statistical variations in the input stimuli. The unlicensed systems (Secondary users) are allowed to use unused spectrum of licensed systems (Primary users) [2].

Figure 1 shows the dynamic spectrum access in Cognitive radio

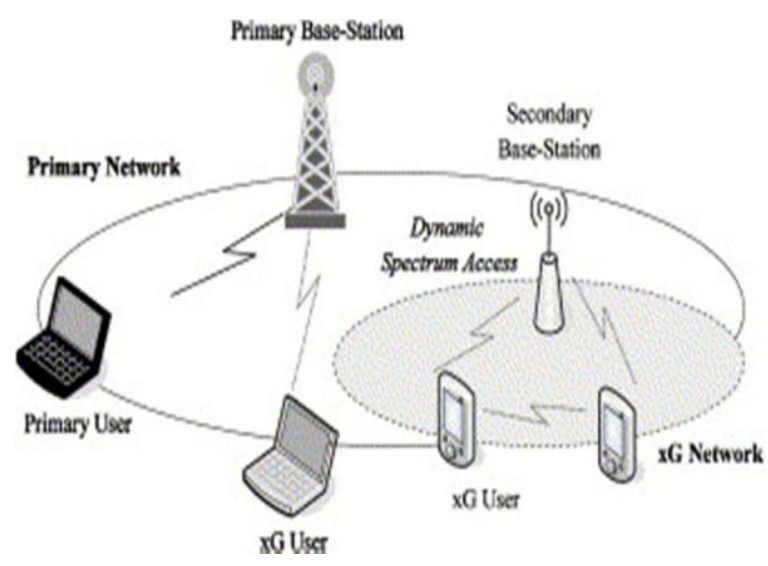

Fig. 1: Dynamic spectrum access

The basic Cognitive cycle performs the following tasks.

- Spectrum Sensing.

- Spectrum Management.

- Spectrum Sharing.

- Spectrum Mobility.

Spectrum Sensing is to recognize the availability of licensed users and unutilized frequency bands i.e white spaces in those licensed bands.

Spectrum Management is to recognize how long the secondary users can use those white spaces.

Spectrum Sharing is to allocate the white spaces (spectrum- hole) equally among the secondary users.

Spectrum Mobility is to maintain unbroken communication during the transition to better spectrum [3] [4]. 
In terms of occupancy, sub bands of radio spectrum may be classified as [5]:

a) White spaces: One which is completely empty except for noise due to natural or artificial sources.

b) Gray spaces: These are partly occupied by interfering signals.

c) Black spaces: One which is fully occupied by communication signals, interfering signals and noise.

Figure 2 shows White spaces and used frequencies in the licensed spectrum

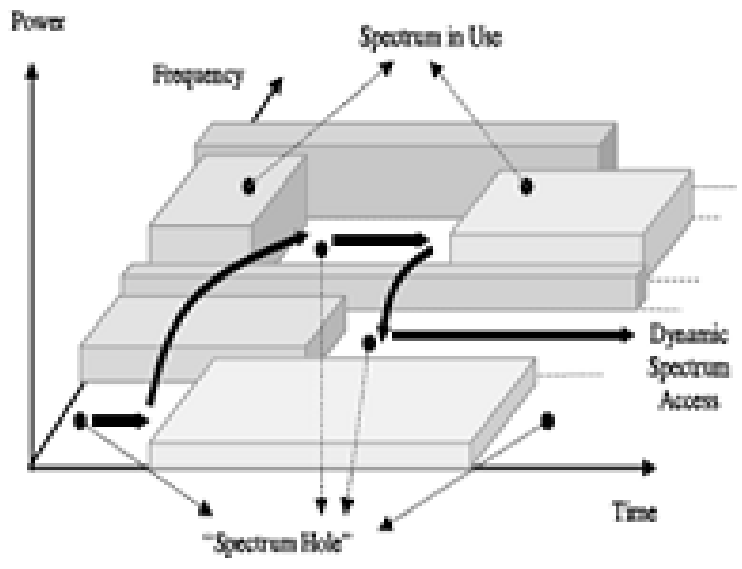

Fig.2: White spaces and used frequiencies

Benefits of Cognitive Radio:-

- Spectrum efficiency:- The future requirement of spectrum will be met and this is the basic reason for implementing CR.

- Improved Quality of service:- From the user's perspective the suitability, availability and reliability will be enhanced.

- Higher bandwidth services:- Necessity of Multimedia broadcast and multicast service is constantly increasing and this will be promoted by implementing CR.

- Future-proofed products:- Without updating a new device by user or manufacturer, CR is able to make changes in services, protocols, modulation, spectrum etc.

- Commercial Exploitation:- CR makes it easier to trade between spectrum and users (Spectrum liberalization). Indeed a business case may exist to become a spectrum broker where by a third party manages the trade between supplier and demander and receives a commission.

- Emergency service communications:- The efforts during major incidents would take advantage a police, fire, ambulance and coastguard would be linked together in one radio with each radio user sensing the spectrum being used by other parties and reconfiguring itself [5].

\section{Spectrum Sensing}

Sensing means to identify free frequency band in the radio environment and plays an important role in $\mathrm{CR}$. The main task of CR is to detect the existence of the primary user by secondary user and to leave the frequency band if the corresponding primary radio emerges in order to prevent interferences to primary users [6] [7].

Basically spectrum sensing technique is broadly classified into two main types. They are: Direct sensing technique and Indirect sensing technique. Frequency Domain is other name for Direct sensing where signal approach is carried out for estimation. Time domain is another name for Indirect sensing where autocorrelation of signal is used to carry out estimation. The classification of Spectrum sensing is given as follows:

\section{Primary transmitter Detection:-}

The detection of the signal from the primary transmitter is based on the received signal at the CR user whether it is present or not [8] [9]. Match filter based detection, Energy based detection, Cyclostationary based detection comes under this category.

\section{Cooperative and collaborative detection:-}

In this information from multiple Cognitive user is incorporated for primary user detection. This 
includes centralized approach or distributed approach.

\section{Interference temperature detection:-}

In this secondary users exist side by side with primary users and secondary users are allowed to transmit at lower power than the primary users. This technique is limited by interference temperature level so that there is no interference. In this approach, CR works in the ultra wide band technology (UWB).

\section{Primary receiver detection:-}

This detection is based primary receiver's local oscillator leakage power.

\section{Spectrum Sensing Schemes:-}

Figure 3 shows the classification of spectrum sensing techniques. Basically spectrum sensing techniques include cooperative systems, non cooperative systems and interference based detection. Non cooperative system further includes energy based detection, match filter detection and cyclostationary detection. We will briefly discuss them one by one and also try to find out their advantages and disadvantages so that each of the technique is properly studied.

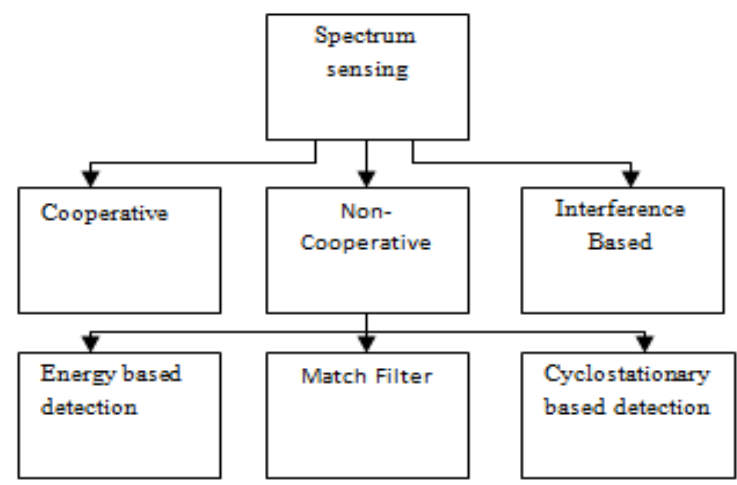

Fig. 3: Classification of Spectrum sensing

Non Cooperative detection:- Basically weak signal from primary transmitter is under observation in this technique. The signal strength generated from the primary user is determined by a cognitive user. Since there is no signaling between primary users and cognitive users, the position of primary receivers are not known to the cognitive users. The fundamental model for transmitter detection can be defined as:-

$\mathbf{X}(\mathrm{t})=\left\{\begin{array}{c}w[n] \\ h s[n]+w[n]\end{array}\right.$

where $x(\mathrm{t})$ is signal received by the cognitive users, $\mathrm{s}(\mathrm{n})$ is transmitted signal of the primary user, $\mathrm{w}(\mathrm{n})$ is Additive White Gaussian Noise(AWGN), $\mathrm{h}$ is amplitude gain of the channel. In this we are going to discuss few transmitter detection techniques.

\section{1) Energy Based detection:-}

This is a most common method of spectrum sensing because of low complexity and computational cost. The receivers do not require any prior knowledge on the primary user's signal. Energy detection can be done by comparing energy of a received signal in a certain frequency band to properly set decision threshold. If the signal energy is greater than the decision threshold, then the frequency channel is said to be busy else the channel is supposed to be free and could be accessed by the CR users [10]. The block diagram for Energy detection technique is shown in the figure 4. Energy Detector has band pass filter which gives the frequency of interest by limiting the bandwidth of received signal, square law device which squares each term of received signal and a summation device which sums all the square values to compute the energy. In this energy detection, energy of an averaged signal is subjected to two hypothetical test functions.

$\mathrm{H} 0$ (PU is absent)

$\mathrm{H} 1$ (PU is in operation)

Under $\mathrm{HO}$

$\mathrm{X}[\mathrm{n}]=\mathrm{w}[\mathrm{n}] ;$ (occurrence of noise only)

Under $\mathrm{H} 1$

$\mathrm{X}[\mathrm{n}]=\mathrm{s}[\mathrm{n}]+\mathrm{w}[\mathrm{n}] ;$ (occurrence of signal with noise) 
Here $\mathrm{n}=0,1,2 \ldots, \mathrm{N}-1, \mathrm{~N}$ shows the index of sample, $\mathrm{w}[\mathrm{n}]$ designate the noise and $\mathrm{s}[\mathrm{n}]$ is the primary signal to detect.

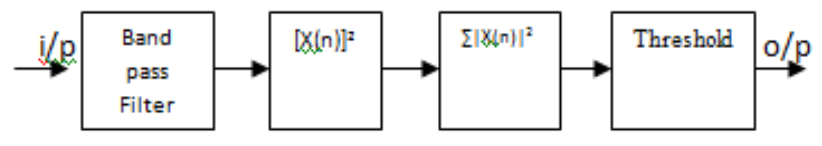

Fig.4: Block diagram of Energy Detector

\section{Advantages of Energy based Detection:-}

- It is having low operating cost and low complexity.

- The probability of making decision is faster. It can cause less delay relative to use.

- It does not require prior knowledge on the received signal.

\section{Disadvantages:-}

- Threshold used depends on the noise variance.

- It cannot differentiate between the interference from the other secondary users sharing the same channel and with the primary user.

- Its performance degrades under low signal-to-noise ratio (SNR) or with noise uncertainty.

- It is inappropriate for spread spectrum techniques like direct sequencing and frequency hopping.

\section{2) Match Filter Detection:-}

Match filter is a linear filter used to increase the signal to noise ratio (SNR) for a given input. This method is optimal in the sense it maximizes the SNR, minimizing the decision errors. The basic need of this detection is the prior knowledge on the received signal such as bandwidth, modulating type and order, operating frequency and pulse shaping. Basically work performed is equal to the correlation in which Match filter which is linear filter correlates the signal with time shifted version and analyze the final output of match filter and predetermined threshold will determine the presence of primary user. The block diagram of the Match filter is shown in the figure 5 .

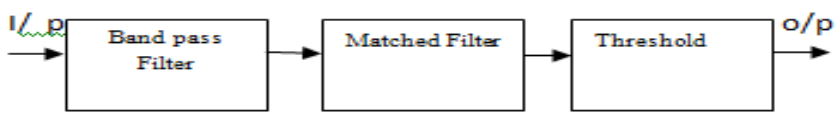

Fig. 5: Block diagram of a Matched filter

Input is fed through band pass filter which will measure the energy around the related band. The output signal of the BPF is convolved with the match filter whose impulse response is same as the reference signal. The output of match filter is compared with the threshold to detect the presence or absence of primary user.

The operation of the Matched filter is given below:

$$
\mathrm{Y}[\mathrm{n}]=\mathrm{h}[\mathrm{n}-\mathrm{k}] \mathrm{x}[\mathrm{k}]
$$

Where $\mathrm{x}$ is unknown signal convolved with the ' $\mathrm{h}$ ', the impulse response of the matched filter that is matched to the reference signal for maximizing SNR.

\section{Advantages:-}

- This technique requires shorter sensing time for certain probability of false alarm or probability of detection.

- Matched filter detection performs well in stationary Gaussian noise when the CR user has the information of the primary user signal.

\section{Disadvantages:-}

- Matched filter detection requires the exact synchronization and prior knowledge of primary user signal.

- Implementation complexity of sensing unit is impractically large since cognitive radio user need receivers for all signal types.

- Since various receiver algorithms need to be executed for detection, it results in large power consumption. Moreover this technique is accomplished when licensed users are cooperating. 


\section{3) Cyclostationary feature detection:-}

This process basically depends on periodicity or statistics of mean or autocorrelation of the signal that vary periodically over time. This periodicity is used in received signal to represent the existence of the primary users [11] [12]. The cyclic correlation factor (SCF) is the key point for detecting the primary user signal. Block diagram of Cyclostationary based detection is shown in the figure 6.

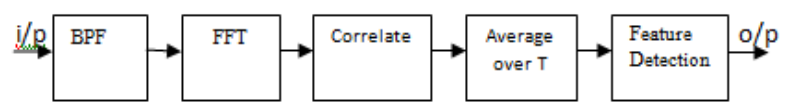

Fig. 6: Block diagram of cyclostationary detection.

The cyclostationary detection is performed as given:-

Firstly cyclic autocorrelation function of observed signal $\mathrm{x}(\mathrm{t})$, is calculated, $R_{x}^{\alpha}(\tau)$, as

$R_{x}^{\alpha}(\tau)=$

$$
\lim _{T \rightarrow \infty} \frac{1}{T} \int_{-T / 2}^{T / 2} x\left(t+\frac{\tau}{2}\right) x\left(t-\frac{\tau}{2}\right) e^{-j 2 \pi \alpha t} d t
$$

Then, the discrete Fourier transformation of the cyclic autocorrelation function (CAF) can be computed to obtain the spectral correlation function (SCF) as:

$$
S_{x}^{\alpha}(f)=\int_{-\infty}^{\infty} R_{x}^{\alpha}(\tau) e^{-j 2 \pi f \tau} d \tau
$$

Spectral correlation function $S_{x}^{\alpha}(f)(\mathrm{f})$ is also called cyclic spectrum, which is a two-dimensional function in terms of frequency and cyclic frequency. Finally, the detection is completed by searching for the unique cyclic frequency corresponding to the peak in the SCF plane. However the boundaries cannot be accurately determined with this method. Hence this technique combined with boundary detection is often used for developing algorithms.

\section{Advantages:-}

- Signals with low SNR can be detected with cyclostationary feature detection.

- It can differentiate between noise energy from received signal energy.

- Cyclostationary method can be used to find out the type of modulation scheme used by the primary user signal.

\section{Disadvantages:-}

- This method requires longer observation time and high cost also because it is computationally complex.

- The detection performance degrades due to the poor estimate of cyclic spectral density.

\section{Cooperative Sensing:-}

Due to the absence of signaling between the primary users and cognitive users, the positions of the primary receivers are not known to the cognitive users in the primary transmitter detection. As a result cognitive radio should depends on the weak primary transmitter signals based on the location of the cognitive users. Also there is no interaction between primary network and cognitive network as they are physically separated from each other [13] [14]. Thus, the cognitive user cannot avoid interference due to the absence of the primary receiver information in shown in figure 7.

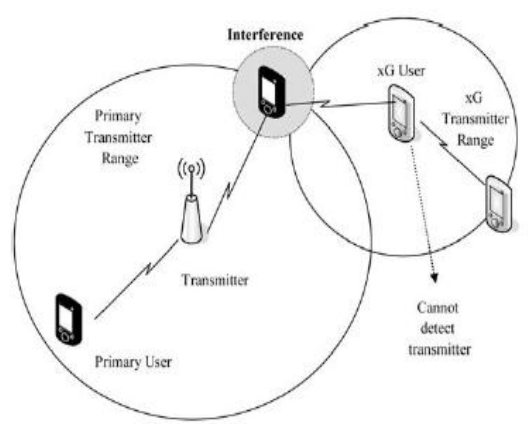


Fig.7: Showing Interference

Also there is hidden-terminal problem associated with the primary transmitter detection because of shadowing shown in figure 8. Moreover Secondary users may be shadowed away from the primary user's transmitter but there may be primary receivers close to the secondary users that are not shadowed from the primary transmitter. As a result, if a secondary user transmits, it may interfere with the primary receiver's reception. To overcome these problems, we need Cooperative sensing. Cooperation allows us to achieve this robustness without drastic requirements on individual radios. Cooperative detection refers to spectrum sensing methods where information from multiple cognitive users is incorporated for primary user detection. Cooperative detection for spectrum sensing can be performed in one of the following ways: centralized server based detection, distributed detection and external detection. We will discuss these one by one.

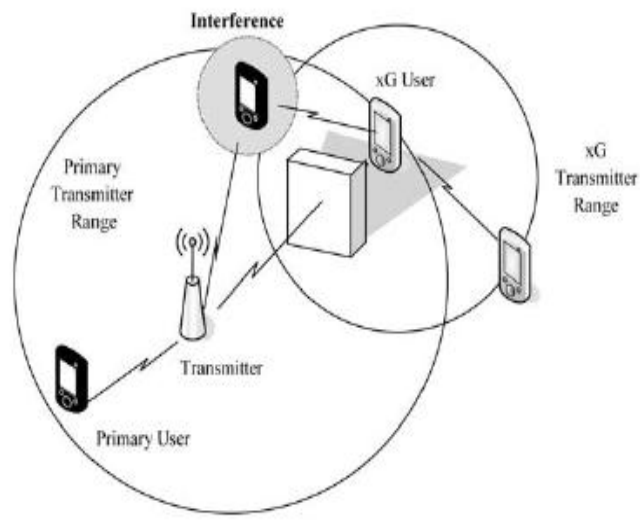

Fig.8: Hidden-terminal problem

\section{Centralized Sensing:-}

The aim of the Centralized sensing is to reduce the fading effects of the channel and increase detection achievement. In this we have Cognitive Radio controller that act as a server, collects all sensed information associated to spectrum occupancy from the CR devices, adding the available information centrally and the broad casts the available information associated to the spectrum status to all CR users. Figure 9 shows the schematic representation of the centralized sensing.

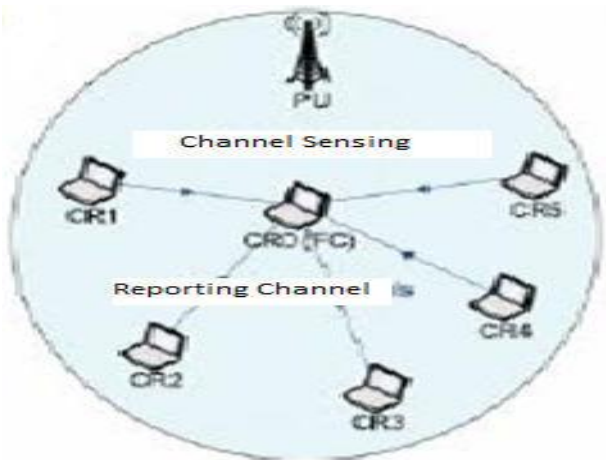

Fig.9: Centralized Sensing

\section{Distributed Sensing:-}

Distributed sensing is more advantageous than centralized sensing because there is no need of backbone infrastructure and has reduced cost [15]. In this, cognitive nodes share information among each other but they make their own decisions as to which part of the spectrum they can use. The individual CR users need to have installed individual sensing unit on them. In this approach cognitive radio CRs operate in distributed cooperative manner; that divide CRs into groups, each of which select the node with the best reporting channel gain as a fusion node. Based on maintained energy detection, CRs performs the local sensing and forward their binary detection decision to fusion node where the processing and fusion of local spectrum observation for candidate nodes is made. The Distributed Cooperative Sensing is modeled with a standard parallel fusion network. A schematic representation of distributed cooperation is illustrated in Fig. 10; its group decision is calculated by the fusion node, sends the result to the fusion centre which computes the global decision from the output. 


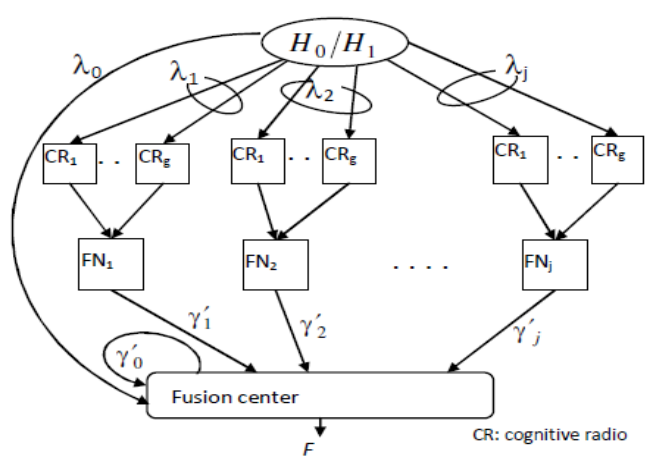

Fig. 10: Distributed Sensing

\section{External Sensing:-}

This technique is similar to centralized server based approach, however, unlike the centralized server based detection, the external agent is equipped with sensing capability with spectrum sensors which performs the sensing and broadcast the channel occupancy information to cognitive radios. The main advantages of external sensing are overcoming hidden primary user problem and the uncertainty due to shadowing and fading. Also the spectrum efficiency is increased as the CR does not spend time in sensing. The power consumption problem of internal sensing can also be addressed because the sensing network does not need to be mobile and not necessarily powered by batteries. Moreover this method is efficient in terms of time since CR users do not spend time detecting primary network as the spectrum sensing is performed by the external agent.

\section{Advantages of Cooperative Sensing}

Hidden node problem is significantly reduced by using a cooperative sensing system because greater number of receivers will be able to build up a might more accurate picture of the transmissions in the area. There is increase in agility because increase in the number of spectrum sensing nodes by cooperation enables the sensing to be more accurate and better options for channel moves to be processed, resulting in increased agility. Cooperative sensing results in reduced false alarms by having multiple nodes performing the spectrum sensing results in more accurate channel signal detection and this reduces the number of false alarms.

\section{Disadvantages:-}

Cooperative sensing too has disadvantages which include Limited Bandwidth, Short Timescale, Scalability.

\section{B) Interference Based Detection:-}

There are two methods proposed for interference based detection.

(a) Interference Temperature Management

(b) Primary Receiver Detection.

\section{(a) Interference Temperature Management:-}

Usually, through the radiated power and location of the individual transmitters, interference can be controlled at the transmitter [16]. However at the receivers, interference actually takes place as shown in the figure 7. For this a new model for measuring interference, referred to as "interference temperature", has been proposed. According to this model, the signal of a radio station is designed to operate in a range at which the received power approaches the level of the noise floor. The noise floor increases at various points within the service area as additional interfering signals appear, as indicated by the peaks above the original noise floor as shown in fig.11. 


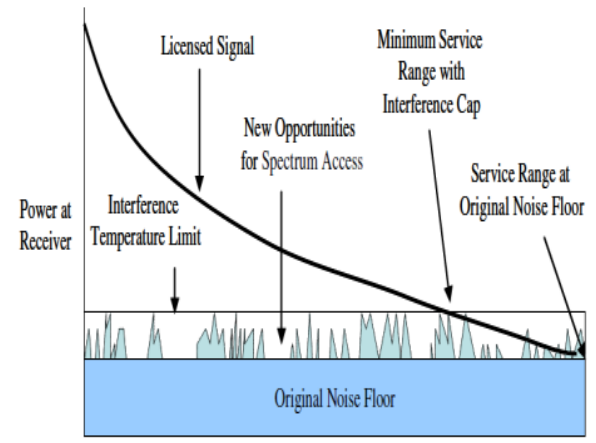

Fig. 11:

The interference temperature is defined to be the RF power measured at a receiving antenna per unit bandwidth. The concept for this metric, first, the interference temperature at a receiving antenna provides an accurate measure for the acceptable level of RF interference in the frequency band of interest; any transmission in that band is considered to be "harmful" if it would increase the noise floor above the interference temperature threshold. The interference temperature model control interference at the receiver through the interference temperature limit, which is defined by the amount of new interference that the receiver could bear. . However, there exist some limitations in measuring the interference temperature. The difficulty of this model lies in accurately determining the interference temperature limit. Secondary CR users cannot transmit their data with higher power because of imposed low transmit power and interference temperature limit even if the licensed system is completely idle for given time and location. This can be seen as another disadvantage of this approach.

\section{(b) Primary receiver detection:-}

It has been suggested as a method to detect primary user by mounting a low cost sensor node close to a primary user's receiver in order to detect the local oscillator (LO) leakage power emitted by the RF front end of the primary user's receiver which are within the communication range of CR system users. The local sensor then reports the sensed information to the CR users so that they can identify the spectrum occupancy status. The spectrum opportunities to operate CR users in spectrum overlay can also be identified using this method.

\section{Comparison Of Various Spectrum Sensing Techniques.}

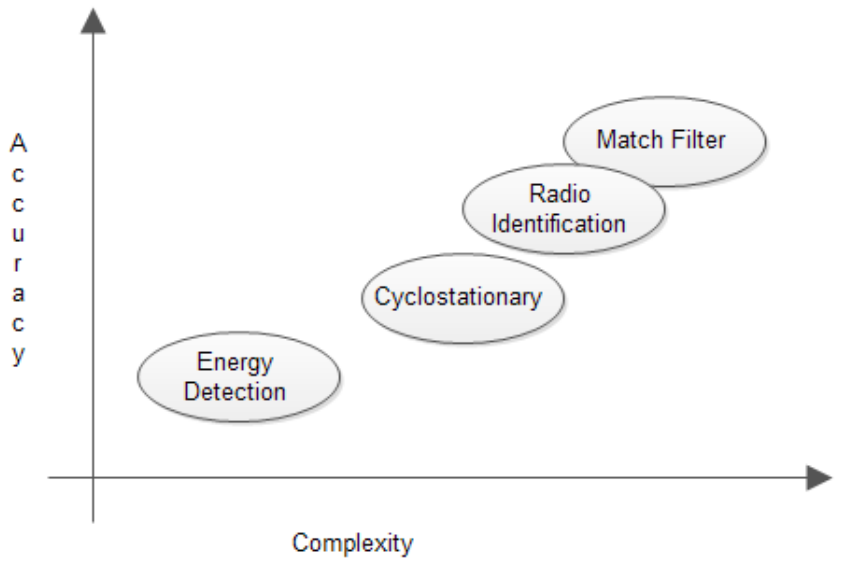

Fig.12: Comparison of spectrum sensing techniques.

We studied various Spectrum sensing techniques. Each has their own merits and demerits. A precise comparison is given here. We note that the matched filtering is giving the highest accuracies with high complexity. It also has the low computational cost. High complexity is because of the implementation of many matched filters in cognitive radio devices for spectrum sensing in wide band regime. It also requires a priori knowledge of the primary user. On the other hand Energy detection is least accurate and least complex since we do not need any special kind of filters. Others are in the middle in terms of accuracy and complexities. Cyclostationary feature detection depends on periodicity or statistics of mean or autocorrelation of the signal that vary periodically over time. It requires partial information of the primary user. However, it is robust to interference. It is very difficult to differentiate between the noise and the desired signal in case of the energy detector. Due to which there is an increased chance of false alarms in energy detection. The performance of the 
energy detector based sensing is limited because of the increased sensing time. There is also a minimum SNR below which signal cannot be detected. Energy detection does not stand good when the noise may not be stationary and its variance may not be known. The advantage of energy detection method is that it does not need any a priori information and it has Low computational cost. Signals with low SNR can be detected with cyclostationary feature detection. It can differentiate between noise energy from received signal energy. Cyclostationary method can be used to find out the type of modulation scheme used by the primary user signal. However there are problems associated with these methods which include Interference due to the lack of the primary receiver's information, Hidden terminal problem due to shadowing. In order to overcome these problems we use Cooperative sensing. Cooperative detection method is more accurate. But the complexity is increased in case of cooperative detection. Traffic overhead is also increased and there is the need for a control channel. To overcome the problems of fading and hidden terminal problem because of shadowing and other blockings, we may use primary receiver detection method. This approach relies on the energy detection technique. The advantage of primary receiver detection over the transmitter detection is its ability to locate the primary user, locate the exact primary channel in use and the high probability to find the free spectrum even in the high density of the primary receivers. However, the disadvantage lies in the need for a highly sensitive energy detector, high cost of the architecture, the near-far problems etc. Another approach for spectrum sensing is the interference temperature based detection. The basic idea behind the interference temperature management is to set up an upper interference limit for given frequency band such that the CR users are not allowed to cause harmful interference while using the specific band in specific area. Mainly, CR user transmitters control their interference by regulating their transmission power based on their locations with respect to primary users. The difficulty of this model lies in accurately determining the interference temperature limit. However, the main disadvantage of this method is that the cognitive radio users can not transmit their data with higher power even if the licensed system is completely idle since they are not allowed to transmit with higher than the preset power to limit the interference at primary users. The cognitive radio users in this method are required to know the location and corresponding upper level of allowed transmit power levels. This is also another disadvantage of this method.

\section{Issues In Spectrum Sensing}

For spectrum sensing to function properly, there exist many challenges that need to be examined which includes [17]

\section{a. Channel Uncertainty:-}

There will be uncertainties in the received signal strength which will lead to wrong interpretation because of fading and shading of the channel. To overcome this, Cognitive Radios must have high sensitivity so that he can differentiate between faded primary signal and a white space. A single cognitive radio cannot give high sensitivity if the fading is severe, to handle this we go for a set of cognitive radios which share their local measurements and collectively decide on the occupancy state of a licensed band.

\section{b. Aggregate Interference Uncertainty:-}

If multiple Cognitive Radios are operating same in same licensed band which will lead to spectrum sensing will be affected by uncertainty in aggregate interference. Even though the primary user is out of interference range this uncertainty may lead to wrong detection so this uncertainty will create a need of more sensitive detector.

\section{c. Security-}

There are various security concerns associated with the cognitive radio. A selfish user in cognitive radio masquerades as primary user. Hence, it can take advantage of that and keeps the spectrum occupied when there is no legitimate primary user in the spectrum. We call this as primary user emulation (PUE) attack to denote this attack. To thwart such attacks, a transmitter verification scheme that can differentiate between legitimate incumbent signal transmitters and secondary signal transmitters (emulating incumbent signal transmitters) is used. In this scheme mainly two techniques are used:- a) Distance Ratio Test (DRT) b) Distance Difference Test (DDT). The Distance Ratio Test (DRT) uses received signal strength (RSS) measurements that are obtained from a pair of verifiers to verify the transmitter's location. The second technique i.e. Distance Difference Test (DDT), utilizes the phase difference of the primary user's signal observed at a pair of verifiers to verify the transmitter's location. A more challenging task is to invent methods that can prevent these attacks. One such method is to employ Public key ciphers used as digital signatures [18]. The primary user encrypts its identification with its private key that is known to it only and appends the encrypted value (signature) to its transmission. All the secondary users, scan for the signature during the sensing period and the signatures from various secondary users are consolidated at the associated secondary base station. The verification of these signatures is done by secondary base station. Since only the primary user knows its private key, a malicious 
secondary user could not have produced a valid signature. Some of the advantages of the proposed scheme are that it is light weight, the key management is simple in it and it can detect accidental asynchrony in secondary users. But this method can only be used in the digital domain not with the analog primary users.

\section{d. $\quad$ Sensing Interference Limit:}

There are two factors for this issue that is when an unlicensed user may not know exactly the location of the licensed receiver which is required to compute interference caused due to its transmission and the second reason is that the transmitter may not be aware of the receiver if a licensed receiver is a passive device. So while calculating sensing interference limit these factor need attention.

\section{Conclusion}

In this paper need and origin of cognitive radio spectrum has been discussed. There are various spectrum sensing methods which one can use for sensing by the use of cognitive radio network which is being discussed one by one in this paper. Merits and demerits of each spectrum sensing technique are also mentioned in the paper. One method proves to be efficient under some conditions and other method seems to be efficient under some other conditions, so there is still a lot of research work left to be done to get the optimal sensing method. Apart from that there are various challenges associated with the practical implementation of cognitive radio which need to be addressed and resolved very properly.

\section{References:-}

[1]. J. Mitola III, "Cognitive Radio for Flexible Multimedia Communications", Mobile Multimedia Communications, 1999, IEEE International Workshop on, pp. 3-10, 1999.

[2]. Ekram Hossain, Dusit Niyato Zhu Han, "Dynamic Spectrum Access and Management in Cognitive Radio Networks", Cambridge University Press, 2009.

[3]. Tevfik Yucek and Huseyin Arslan (2009), “A Survey of Spectrum Sensing Algorithms for Cognitive Radio Applications”, IEEE Communication Surveys \& Tutorials, Vol.11, No.1, First Quarter 2009, pp 116-130.

[4]. Ian F. Akyildiz, Won-Yeol Lee, Mehmet C. Vuran and Shantidev Mohanty, "A Survey on Spectrum Management in Cognitive Radio Networks", Georgia Institute of Technology, pp. 1-2.

[5]. Y. Yuan, P. Bahl, R. Chandra, P. A. Chou, J. I. Ferrell, T. Moscibroda, S. Narlanka, and Y. Wu, "Cognitive Radio Networks Over White Spaces", in Proc. IEEE Int. Symposium on New Frontiers in Dynamic Spectrum Access Networks, 2007, Dublin, Ireland, pp 416-427.

[6]. Lakshmi Thanayankizil, Aravind Kailas, "Spectrum Sensing Technique (II): Receiver Detection and Interfer-ence Management", 2008.

[7]. S. Haykin, D. J. Thomson, and J. H. Reed, (2009) "Spectrum sensing for cognitive radio," Proceedings of the IEEE, vol. 97, no. 5,pp. 849-877.

[8]. B. Wild and K. Ramchandran, "Detecting Primary Receivers for Cognitive Radio Applications" in proceeding of IEEE Dynamic Spectrum Access Networks, DySPAN 2005, November 2005.

[9]. Digham, M. Alouini, M. Simon, "On the energy detection of unknown signals ove fading channels", Department of Electrical and Computer Engineering, University of Minnesota, USA.

[10]. Rahul Tandra, Anant Sahai, "Fundamental limits on detection in low SNR under noise uncertainty" University of California, Berkeley.

[11]. K. Kim, I.A. Akbar, K.K. Bae, J.-S. Um, C.M. Spooner, and J.H. Reed, "Cyclostationary approaches to signal detection and classification in cognitive radio" in Proceedings of IEEE Symposium on New Frontiers in Dynamic Spectrum Access Networks, Dublin, Ireland, April 2007.

[12]. Subhashri G. Mohapatra, Ambarish G. Mohapatra, Dr. S. K. Lenka, "Performance evaluation of cyclostationary based spectrum sensing in cognitive radio network" IEEE, 2013, pp 90-97.

[13]. Jayakrishnan Unnikrishnan and Venugopal Veeravalli, "Cooperative Sensing for Primary Detection in Cognitive Radio", IEEE Journal of selected topics in signal processing, Vol. 2, No. 1, February 2008.

[14]. Shridhar Mubaraq Mishra, Anant Sahai and Robert W. Brodersen, "Cooperative Sensing among Cognitive Radios" School of Electrical Engineering and Computer Science University of California, Berkeley, California.

[15]. P. Qihang, Z. Kun, W. Jun, and L. Shaoqian, "A distributed Spectrum Sensing Scheme Based on Credibility and Evidence Theory in Cognitive Radio Context", in Proc. IEEE Int. Symposium on Personal, Indoor and Mobile Radio Commun., 2006, Helsinki, Finland, pp 1-5.

[16]. T. C. Clancy, "Formalizing the interference temper-ature model", J. Wireless Commun. Mobile Comput.2007, vol. 7, No. 9, pp1077-1086.

[17]. D. Cabric, S. Mishra, and R. Brodersen, "Implem-entation Issues in Spectrum Sensing for Cognitive Radios", in Proc. Asilomar Conference on Signals Systems and Computers, 2006, vol.1, Pacific Grove, California, USA, pp 772-776.

[18]. C. N. Mathur and K. P. Subbalakshmi, "Digital signatures for centralized DSA networks," in First IEEE Workshop on Cognitive Radio Networks, Las Vegas, Nevada, USA, Jan. 2007. 